\title{
Variabilité saisonnière et intra-saisonnière de la pluviométrie en milieu forestier dans le Sud-ouest centrafricain
}

\author{
Jean-Marie Djebata ${ }^{1}$, Cyriaque R. Nguimalet ${ }^{2}$, and Pierre Camberlin ${ }^{3}$ \\ ${ }^{1}$ Ecole Normale Supérieure de Bangui, Bangui, République Centrafricaine \\ ${ }^{2}$ Département de Géographie, Faculté des Lettres et Sciences Humaines, \\ B.P. 1037, Bangui, République Centrafricaine \\ ${ }^{3}$ Département de géographie/Centre de Recherches de Climatologie, UMR 6282 Biogéosciences, \\ Université de Bourgogne Franche-Comté, Dijon, France \\ Correspondence: Cyriaque R. Nguimalet (cyrunguimalet@gmail.com) and Jean-Marie Djebata \\ (djebata30@gmail.com)
}

Published: 16 November 2021

\begin{abstract}
Résumé. Ce travail présente la variabilité intra-saisonnière de la pluviométrie dans le Sud-ouest centrafricain. Les données pluviométriques journalières utilisées couvrent la période 1981-2017. Elles ont été extraites aux points de grilles les plus proches des quatre stations représentatives de la zone d'étude (Bangui, Berberati, Boukoko et Nola) sur la base de données CHIRPS (Climate Hazards Group InfraRed Precipitation with Station data). Un contrôle de qualité de ces données a été effectué à partir d'une inter-comparaison entre les produits d'estimations et les données in-situ sur différentes sous-périodes : 1998-2011 à Nola, 1998-2012 à Berberati, 1998-2014 à Bangui et 2002-2017 à Boukoko. Le coefficient de corrélation entre les données CHIRPS et les observations au pas annuel est faible à Bangui $(r=0,46)$, moyen à Nola $(r=0,57)$ et Berberati $(r=0,60)$, et bon à Boukoko $(r=0,73)$. Les dates de début de la saison de pluies varient entre le 13 février et le 2 avril et celles de fin entre le 31 octobre et le 4 décembre. Des épisodes secs et humides sont mis en évidence dans le Sud-ouest centrafricain. A Bangui et Berberati, la période du 15 au 22 mai 1999 a été sèche soit 23 jours sans pluie. A Boukoko et Nola, la période du 26 juin au 24 juillet de la même année était caractérisée par des épisodes secs. Ces résultats établissent que la répartition intra-saisonnière des pluies n'est pas uniforme dans le Sud-ouest centrafricain.
\end{abstract}

\section{Introduction}

La connaissance sur l'évolution du climat est devenue l'un des défis majeurs des pays en développement, du fait des impacts négatifs et probables du climat sur l'homme et les écosystèmes (Abossolo et al., 2015).

Le système climatique est sans équivoque et, depuis les années 1950, beaucoup de changements observés sont sans précédent (GIEC, 2014). L'Afrique fait partie des régions du globe les plus vulnérables aux effets des changements climatiques en raison de son faible niveau de développement économique et de sa faible capacité de résilience.
En Afrique centrale, la République Centrafricaine (RCA) n'est pas épargnée de l'impact de la variabilité pluviométrique sur son économie. Ainsi, dans le Sud-ouest du pays, les populations du secteur forestier ont constaté que les pluies deviennent de plus en plus imprévisibles et varient considérablement d'une année à une autre. Les saisons pluvieuses sont parfois marquées par des épisodes secs et des sécheresses intercalaires.

De nombreux travaux ont permis de comprendre les modalités et facteurs de la variabilité pluviométrique en Afrique de l'Ouest, mais ces travaux restent beaucoup plus rares en Afrique centrale (Janicot et Sultan, 2001; Ardoin-Bardin, 2004; Camberlin, 2007). En particulier, la variabilité des 


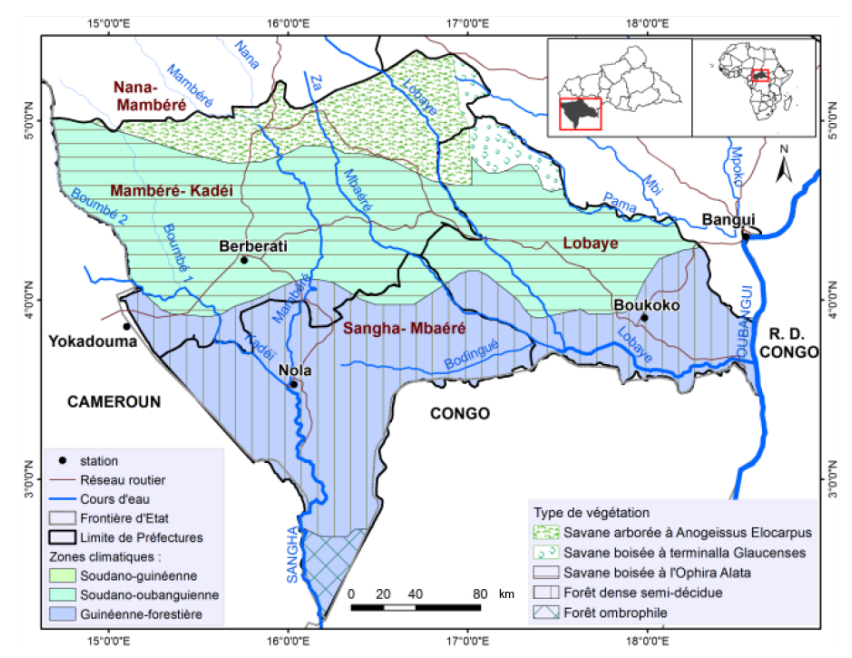

Figure 1. Localisation du Sud-ouest centrafricain. (C) Atlas of the Central African Republic (1984)

pluies à des pas de temps inférieurs à la saison (l'intrasaisonnier) est peu documentée (Camberlin, 2007), exceptés les travaux de Ndjéndolé (2001) dans le Nord-Ouest de la RCA et de Massouangui-Kifouala (2017) en République du Congo.

Toutefois, Zhou et al. (2014) ont détecté à partir de données satellitaires un assèchement de la petite saison pluvieuse (avril-juin) au cours des dernières décennies en Afrique centrale. Il a été aussi récemment démontré que l'intensité et la fréquence des orages avaient augmenté en février (Taylor et al., 2018) et en avril-mai-juin entre 1982 et 2016 dans le bassin du Congo (Raghavendra et al., 2018). Est-ce que les résultats obtenus sur la dynamique saisonnière, voire intrasaisonnière, de la pluviométrie en Afrique centrale ou dans le bassin du Congo sont-ils vérifiables dans le Sud-ouest centrafricain? A cet effet, cette étude examine la variabilité des précipitations au pas de temps journalier et intra-saisonnier dans le Sud-ouest centrafricain.

\section{Présentation de la zone d'étude}

Le Sud-ouest centrafricain couvre une superficie de $94200 \mathrm{~km}^{2}$ sur les $623000 \mathrm{~km}^{2}$ que couvre la RCA, soit $15,12 \%$ du territoire national. Il regorge aussi $41,70 \%$ de forêts denses humides du pays (COMIFAC, 2015). Compris entre $2^{\circ} 13^{\prime}$ et $7^{\circ} 24^{\prime}$ de latitude Nord et entre $14^{\circ} 01^{\prime}$ et $19^{\circ} 02^{\prime}$ de longitude Est, il est limité au nord par la Préfecture de la Nana-Mambéré, au Sud par les Républiques Démocratique du Congo et du Congo, à l'Est par la Préfecture de l'OmbellaMpoko et à l'Ouest par le Cameroun (Fig. 1).

Trois domaines climatiques caractérisent cet espace géographique : la zone guinéenne-forestière plus humide au sud, caractérisée par une pluviosité annuelle supérieure à $1700 \mathrm{~mm}$, la zone soudano-oubanguienne au centre avec
1600 mm et la zone soudano-guinéenne au nord, marquée par un cumul pluviométrique annuel variant de 1200 à $1500 \mathrm{~mm}$ (Fig. 1).

\section{Données et méthodologie}

\subsection{Données}

Deux types de données de pluie au pas de temps journalier et annuel ont été utilisés pour caractériser la variabilité des précipitations : il s'agit des données pluviométriques réellement observées et des estimations de pluies du produit CHIRPS (Climate Hazards Group InfraRed Precipitation with Station data) produits par Earth Resources Observation and Science (EROS), un organisme américain) d'une résolution spatiale de 0,05 . Les données d'observation sont incomplètes dans les quatre stations de la zone d'étude, c'est pourquoi, les données CHIRPS combinant les estimations satellitaires et les observations in-situ, couvrant la période 1981-2017 ont été utilisées. En Afrique centrale, ce produit d'estimation des pluies a été jugé comme l'un des plus fiables à l'échelle interannuelle (Camberlin et al., 2019).

\subsection{Méthodes}

Les données CHIRPS ont été choisies à partir d'une intercomparaison entre les données observées dans les stations principales de la zone d'étude et les points de grille les plus proches, sur différentes sous-périodes : 1998-2014 à Bangui, 1998-2012 à Berberati, 1998-2011 à Nola et 2002-2017 à Boukoko.

Le coefficient de corrélation entre les données CHIRPS et celles observées à l'échelle annuelle est faible à Bangui $(r=0,46)$, moyen à Nola $(r=0,57)$ et fort à Berberati $(r=$ $0,60)$ et Boukoko $(r=0,73)$.

Pour valider le nombre d'événements pluvieux, nous avons confronté le nombre de jours de pluies des deux produits (CHIRPS et Observation) sur les différentes souspériodes mentionnées ci-dessus. Avec CHIRPS, le nombre annuel de jours de pluies est de 184 jours à Bangui, 180 jours à Boukoko et 175 jours à Berberati. Cependant, avec les données d'observation, le nombre de jours de pluies est de 145 jours à Bangui, 140 jours à Boukoko et 138 jours à Berberati. On constate que CHIRPS surestime le nombre d'événements pluvieux, caractérisés par de faibles intensités $(8,1$ à $9 \mathrm{~mm}$ ) contre (10,8 à 14,3 mm) pour les données in-situ.

L'analyse intra-saisonnière de la pluviométrie a reposé premièrement sur la détection des dates de début et de fin de la saison des pluies et la recherche des épisodes secs et humides au sein de la saison des pluies. Dans cette étude, nous avons choisi la méthode simple «d'anomalies cumulées de pluies » basée exclusivement sur les pluies journalières (Barraud, 2017). Avec la méthode d'anomalies cumulées, on a calculé tout d'abord, la moyenne journalière des pluies sur l'ensemble des années. Ensuite, cette moyenne est générale- 

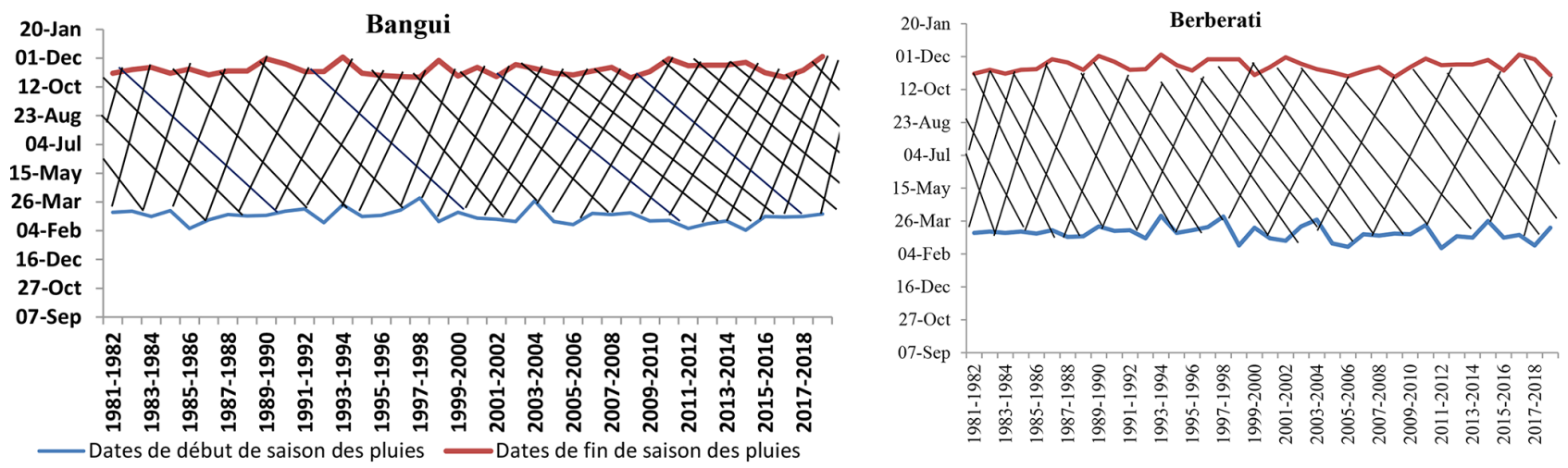

Dates de début de la saison de pluies —Dates de fin de la saison de pluies

Figure 2. Variation interannuelle des dates de début et de fin de saisons des pluies à Bangui et Berberati de 1981 à 2018.

ment affectée d'un coefficient, lequel a permis d'obtenir une valeur de référence nommée (Pref) à partir de laquelle ont été calculées les anomalies journalières des pluies. Pour ce travail, un coefficient de 0,5 a été utilisé pour calculer la valeur de référence (Liebmann et al., 2012). Pour cette étude, la période de référence est comprise entre le 40ème jour (J1) à partir du 9 août et le 330ème jour (J2) et délimite la date de début et de fin de l'analyse. Le principal critère de choix de cette période est d'être centrée sur le cœur de la saison des pluies en débordant largement sur la Saison sèche. A l'intérieur de la période considérée, sont déterminées les dates de début et de fin de la saison des pluies. On calcule année par année, les anomalies cumulées de précipitations journalières. La date de la valeur minimale de ces anomalies cumulées, pour l'année A, est la date de début de saison des pluies de cette année. La date de la valeur maximale de ces anomalies cumulées est la date de fin de saison des pluies. L'utilisation de cette méthode a été justifiée par le fait qu'elle a déjà été appliquée à des zones similaires à celle de l'étude comme l'Amérique du Sud (Liebmann et al., 2008) ou l'Afrique dans son ensemble (Liebmann et al., 2012).

L'analyse interannuelle de la pluviométrie a reposé d'une part, sur une analyse en composantes principales (ACP), qui permet de détecter les structures spatiales de la variabilité (Sanders, 1989), et d'autre part, sur des tests appliqués aux séries centrées-réduites selon la formule proposée par (Lamb, 1983) qui s'écrit :

$X_{i j}=\frac{\left(x_{i j}-\bar{x}_{i}\right)}{\sigma_{i}}$

Où $X_{i j}=$ hauteur de pluie totale pour une station $i$ et une année $j ; \bar{x}_{i}=$ moyenne annuelle de pluie à la station $i$ pendant la période de l'enregistrement; $\sigma_{i}=$ écart type de la série pluviométrique annuelle. Le test de Pettitt (1979) a été utilisé pour la détection des ruptures et tendances climatiques des séries temporelles.

Une analyse en composantes principales a été réalisée pour décrire la structure saisonnière ou la répartition des pluies se- lon la saison DJF, MAM, JJA et SON. La première composante principale de chaque saison, cartographiée sur Google (Climate explorer) à partir des coordonnées géographiques de la zone d'étude (latitude et longitude), de la période de référence (1981-2017) et de l'échelle (saisonnière) de la carte est retenue (Fig. 3) L'évolution des séries temporelles à l'échelle saisonnière a été présentée à partir des données issues des premières composantes principales cartographiées (Fig. 4).

\section{Résultats et discussion}

\subsection{Dates de démarrage et de fin de saison des pluies}

Les dates de début de saison de pluies à Bangui oscillent généralement entre le 5 février et le 2 avril (Fig. 2), et celles de fin de saison de pluies sont comprises entre le 28 octobre et le 3 décembre. A Berberati, les dates de démarrage et de fin de la saison humide sont comprises respectivement entre le 15 février et le 3 avril et entre le 19 octobre et le 3 décembre. Comparativement à la zone guinéenne-forestière (Bangui), la zone soudano-guinéenne (Berberati) connait un retard de 10 jours dans la mise en place de la saison humide.

Cependant, avec les données in-situ, la date de démarrage de saison de pluies se situe entre le 25 février et le 9 mars à Bangui et entre le 10 et le 25 mars à Berberati. La date de fin de saison des pluies est comprise entre le 1 et le 15 décembre à Bangui et entre le 1 et 30 novembre à Berberati. Par rapport aux données d'observation, on constate un début précoce de la saison de pluies avec les produits d'estimation, soit un écart de 20 jours à Bangui et 24 jours à Berberati. A l'inverse, on note un prolongement des dates de la fin de saison de pluies avec les données in-situ qu'avec les produits d'estimation. On note aussi un écart d'environ 33 jours à Bangui et 42 jours à Berberati. 
DJF

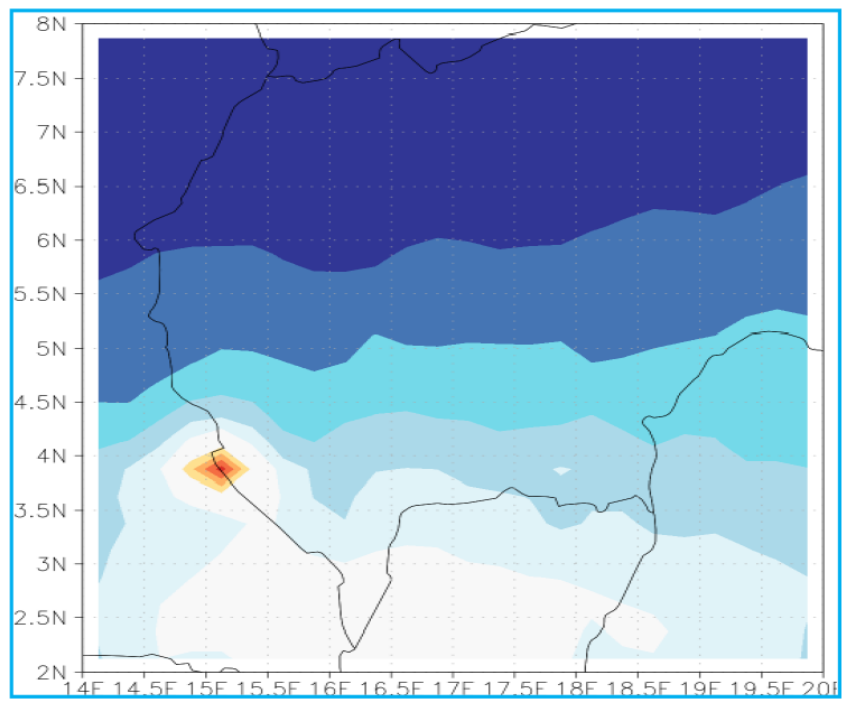

JJA

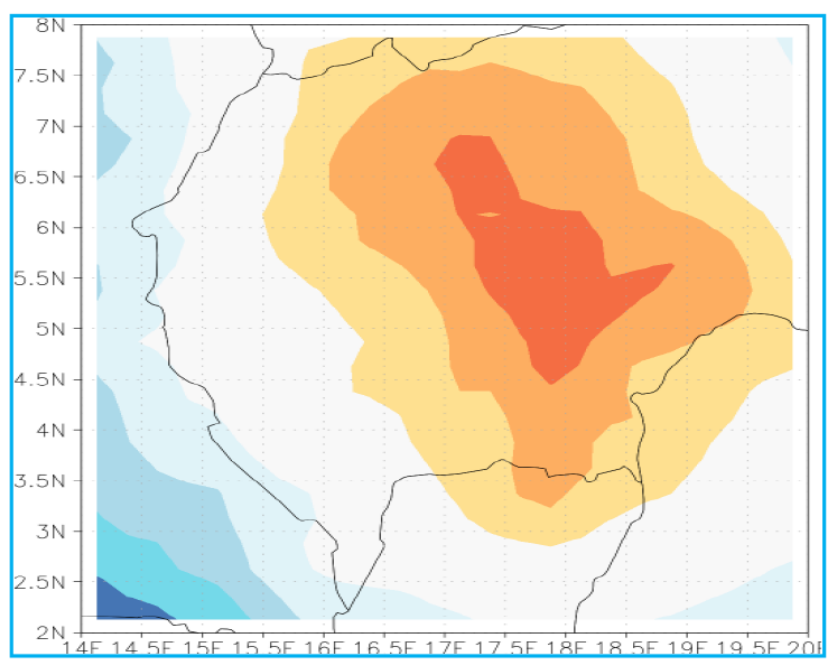

MAM

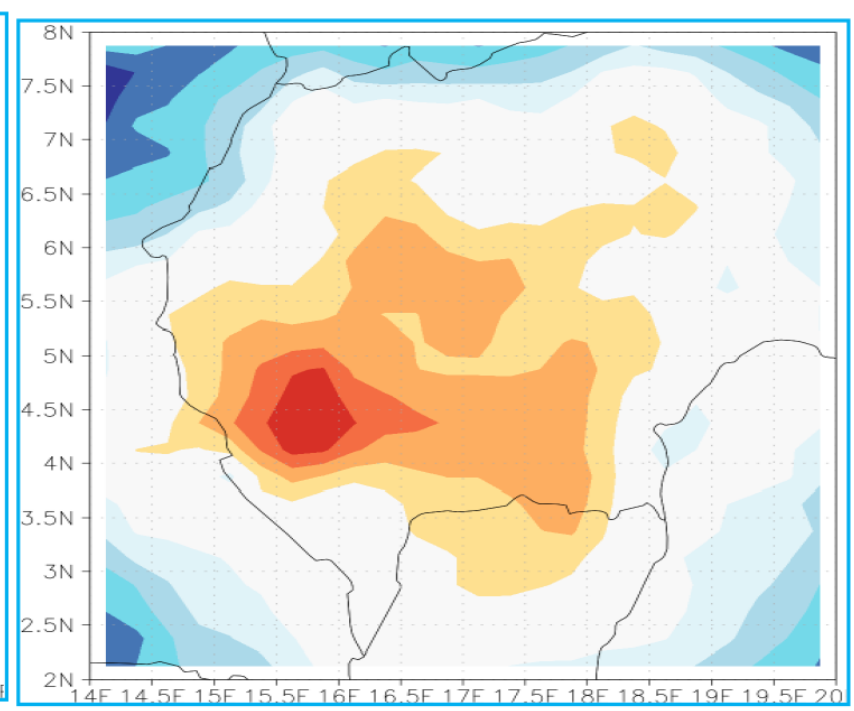

SON

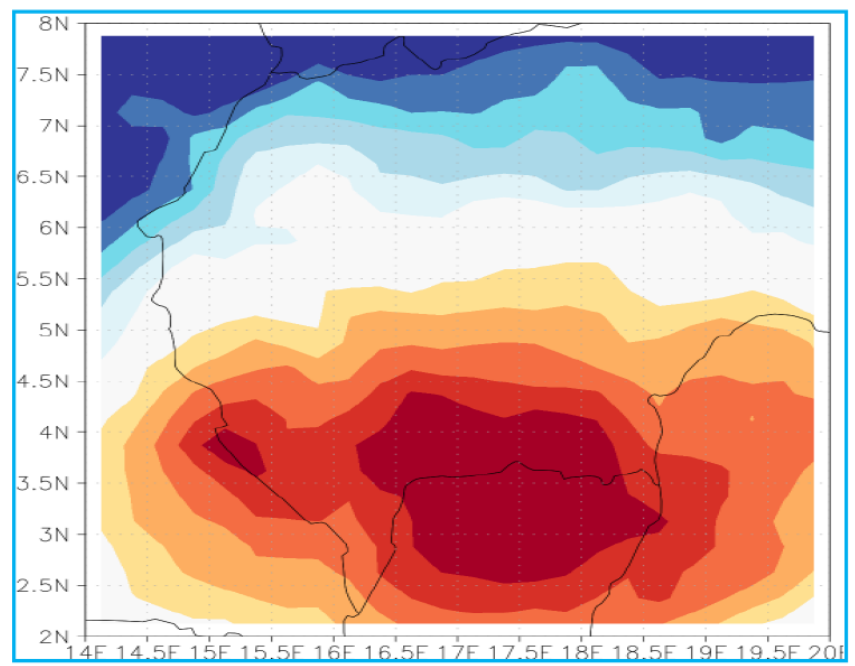

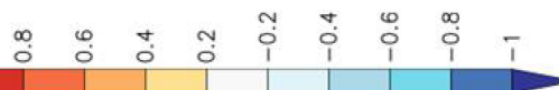

Figure 3. Structures spatiales des pluies associées aux composantes principales de 1981-2017.

4.2 La répartition spatiale des pluies associées aux composantes principales

L'analyse en composantes principales consiste à décrire la variabilité spatiale des pluies à l'échelle saisonnière.

La tendance des pluies est décroissante au trimestre décembre-janvier-février (DJF), croissante en mars-avrilmai (MAM), stationnaire en juin-juillet-août (JJA) et croissante en septembre-octobre-novembre (SON) (Fig. 4).
A l'échelle saisonnière, la répartition des précipitations dans le Sud-ouest centrafricain correspond au mouvement saisonnier de la Zone de Convergence Inter-Tropicale (ZCIT) vers le Nord du pays (Fig. 3). Les moyennes trimestrielles de décembre-janvier-février $(\mathrm{DJF}=66 \mathrm{~mm})$, mars-avrilmai $(\mathrm{MAM}=309 \mathrm{~mm})$, juin-juillet-août $(\mathrm{JJA}=505 \mathrm{~mm})$ et SON $(420 \mathrm{~mm})$ représentent respectivement $5,23 \%$, $23,64 \%, 38,43 \%$ et $32,68 \%$ de la quantité totale des pluies sur la période considérée. 

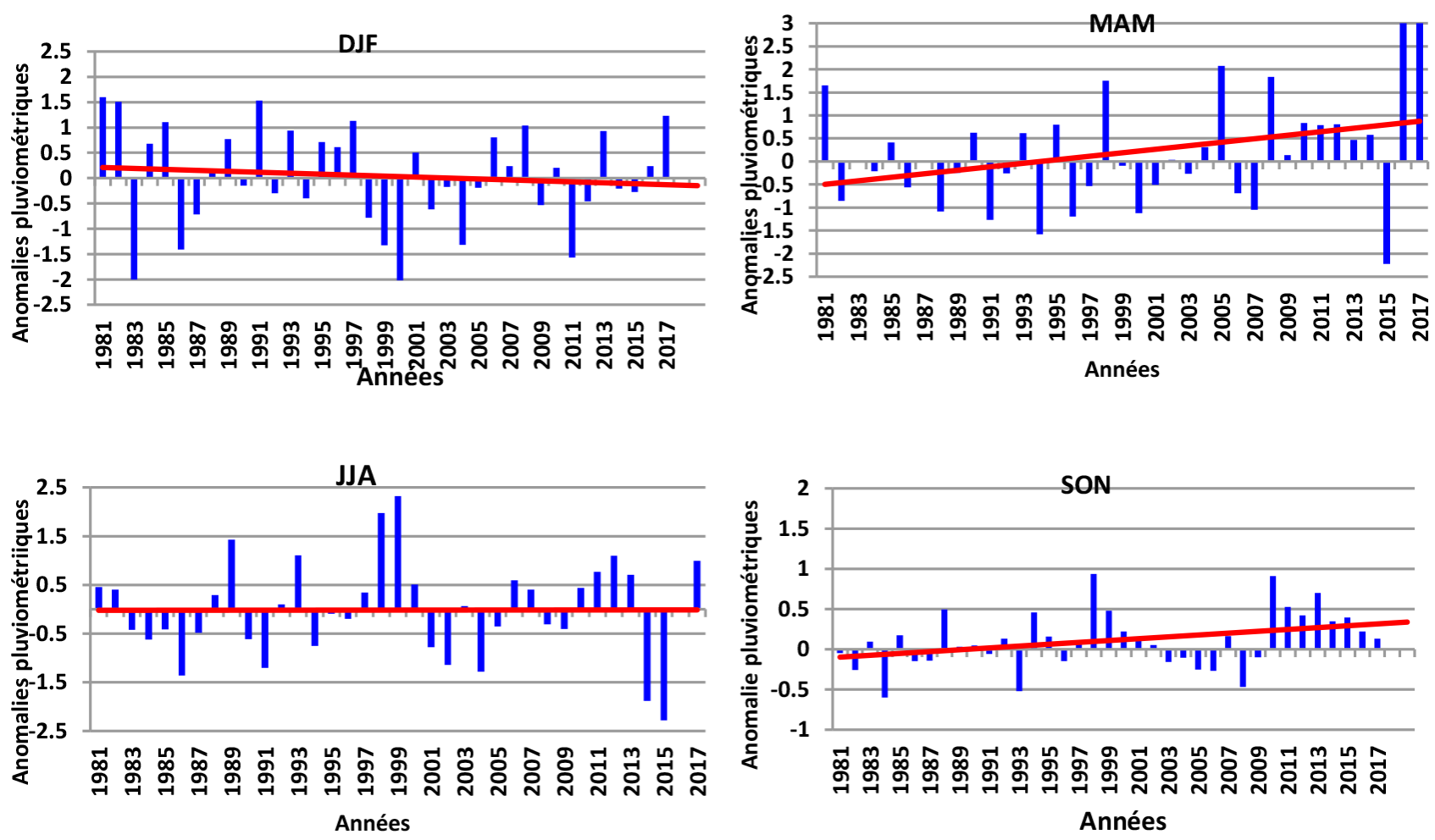

Figure 4. Evolution des séries temporelles associées à chacune des composantes principales cartographiées de 1981 à 2017.

\subsection{Episodes secs et humides au sein de la saison des pluies}

Dans le Sud-ouest centrafricain, la saison de pluies s'étend de mars à novembre, soit 9 mois, marquée par l'alternance des épisodes secs et pluvieux sans cohérence. Deux ou trois épisodes pluvieux sont séparés par une longue période durant laquelle les précipitations journalières peuvent être très faibles (inférieures à $20 \mathrm{~mm}$ ) ou carrément nulles au sein même de la période normalement pluvieuse. Ainsi, une alternance des épisodes secs ( 4 à 6 jours sans pluie) et phases arrosées ( 3 à 5 jours de pluies consécutives) est notée dans les quatre stations représentatives de notre zone d'étude, exclusivement pour l'année 1999 (Fig. 5). En effet, l'année 1999 a été particulièrement humide sur l'ensemble du territoire centrafricain. Elle était caractérisée par des crues et inondations des parcelles agricoles, voire urbaines, dans le Nord du pays où ce phénomène est inhabituel. C'est pour cette raison qu'elle a été choisie ici pour nous permettre de mettre en évidence des épisodes secs au sein de cette année particulièrement humide. En d'autres termes, les sècheresses intercalaires ne s'observent pas seulement pendant les années déficitaires mais également excédentaires sur le plan pluviométrique. On note aussi qu'en 1999, la période du 15 au 22 mai a été sèche à Bangui et Berberati. Les sécheresses intercalaires, surtout en début de la campagne agricole, pourraient être préjudiciables pour les récoltes. En Afrique de l'Est par exemple, Barron et al. (2003) ont estimé qu'un épisode sec survenant durant la phase végétative de floraison peut entraîner une diminution de l'ordre de $72 \%$ à $75 \%$ des rendements de maïs sur sol sableux. A Boukoko et Nola, la période du 26 juin au 24 juillet était caractérisée par des épisodes secs (Fig. 5).

\section{Discussion}

La variabilité intra-saisonnière de la pluviométrie dans le Sud-ouest centrafricain est analysée sur les dates de début et de fin de saison de pluies, la saisonnalité et tendances des précipitations, et les épisodes secs et humides au sein de la saison humide. En domaine guinéen-forestier, les dates de début de saison des pluies oscillent entre le 5 février et le 2 avril. Les dates de fin de saison de pluies sont comprises entre le 28 octobre et le 3 décembre. Cependant, en domaine soudano-guinéen, les dates de démarrage et de fin de la saison humide sont comprises respectivement entre le 15 février et le 3 avril et entre le 19 octobre et le 3 décembre. Ces résultats mettent en évidence une installation plus précoce de la saison humide dans la zone guinéenne-forestière, en marges septentrionales de la grande forêt du bassin congolais, que dans la zone soudano-guinéenne. L'examen des précipitations à l'échelle saisonnière montre que DJF, MAM, JJA et SON représentent respectivement, 5,23\%, 23,64\%, 38,43\% et $32,68 \%$ de la quantité totale des pluies enregistrées sur la période considérée. Nos résultats sont similaires à ceux de 


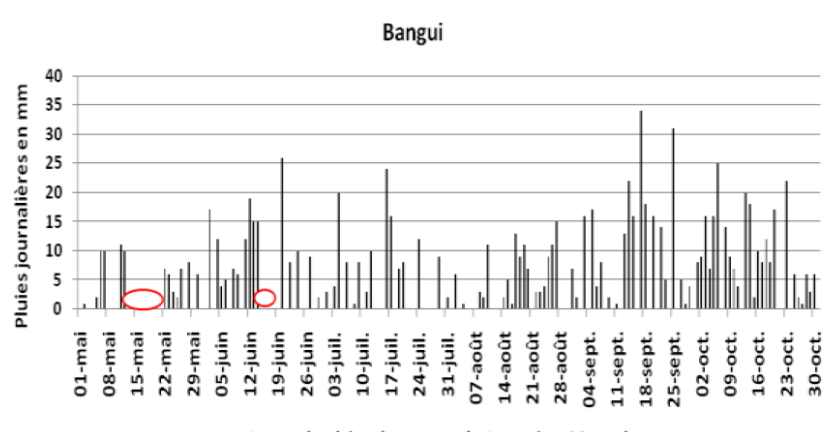

Jours calendaires à compter du 1er mai au 30 cotobre

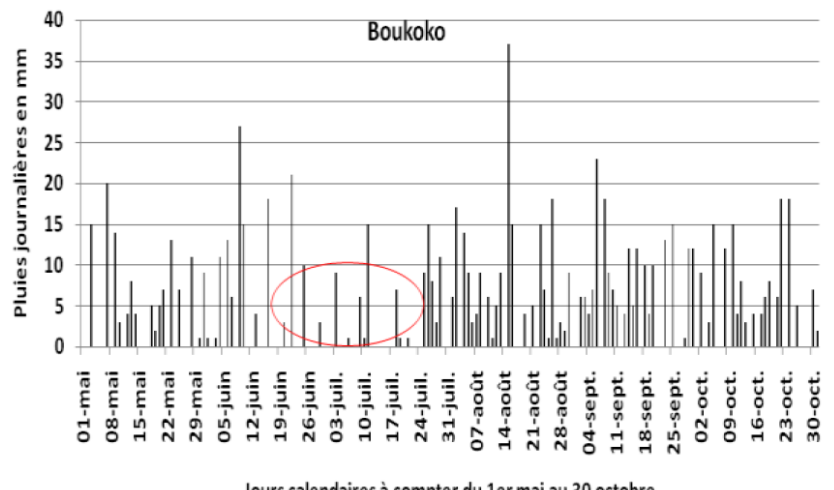

Jours calendaires à compter du 1er mai au 30 octobre

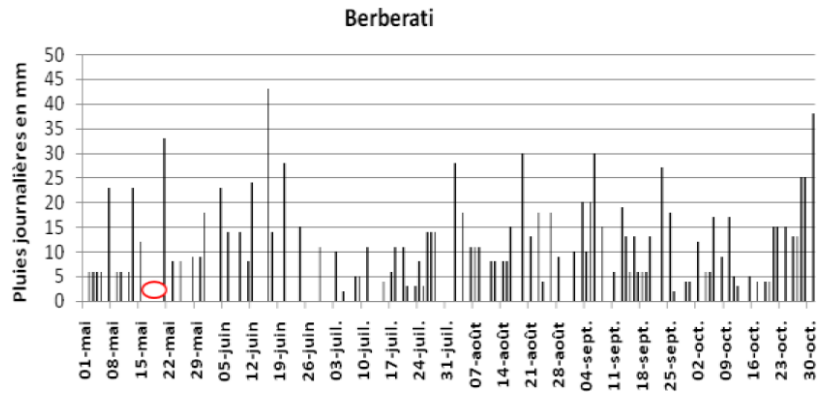

Jours calendaires à compter du 1er au 30 octobre

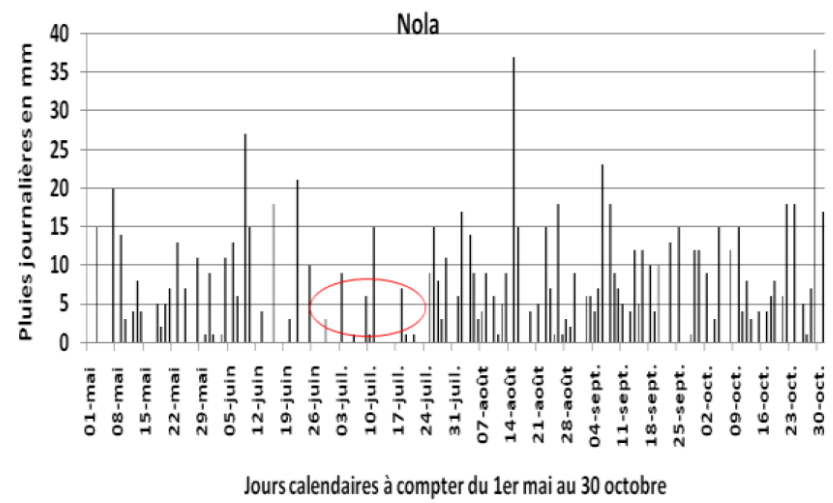

Figure 5. Variation intra-saisonnière des précipitations journalières dans les quatre stations représentatives de la zone d'étude à compter du 1 mai au 30 octobre 1999.

Ndjéndolé (2001). D'après cet auteur, le trimestre JJA constitue le cœur de la saison des pluies en RCA.

Concernant l'évolution des pluies saisonnières, les précipitations sont décroissantes en DJF, stationnaires en JJA, et croissantes en MAM et SON. Ce résultat est similaire à ceux de Raghavendra et al. (2018) et Taylor et al. (2018) qui ont démontré que l'intensité et la fréquence des orages avaient augmenté dans le bassin du Congo en avril-mai-juin et en février entre 1982 et 2016. Par contre, ce résultat ne confirme pas ceux de Zhou et al. (2014) qui ont détecté, à partir de données satellitaires, un assèchement de la petite saison pluvieuse (avril-juin) au cours des dernières décennies en Afrique centrale.

Les fluctuations intra-saisonnières des pluies dans cette zone seraient liées à des facteurs externes, notamment l'Oscillation de Madden-Julian, de l'ordre de 30 à 60 jours, ce qui peut renforcer ou diminuer les précipitations en Afrique centrale (Camberlin, 2007).

\section{Conclusion}

Cette étude révèle un caractère relativement homogène de la pluviométrie dans le Sud-ouest centrafricain, avec néanmoins quelques hétérogénéités locales liées aux conditions géographiques. Elle montre aussi que les dates de début de saison des pluies dans le Sud-ouest du pays oscillent généra- lement entre le 13 février et le 2 avril, et les dates de fin entre le 31 octobre et 4 décembre. Par ailleurs, cette étude indique que comparativement aux données d'observation, la date de début de la saison de pluies est précoce avec les produits CHIRPS. Alors cela ne pose-t-il pas un souci pratique pour la conduite des activités agricoles par exemple, vu qu'une modification du calendrier cultural est notée avec les données d'observation?

Disponibilité des données. Les données pluviométriques utilisées dans ce travail proviennent de l'ASECNA (RCA), et les données CHIRPS disponible sur https://climexp.knmi.nl/ (la date du dernier accès : 14 mars 2019, World Meteorological Organization, 2019).

Collaborateurs. Trois auteurs ont contribué équitablement à la rédaction de ce travail.

Intérêts concurrents. Les auteurs déclarent qu'ils n'ont aucun conflit d'intérêts. 
Clause de non-responsabilité. Publisher's note : Copernicus Publications remains neutral with regard to jurisdictional claims in published maps and institutional affiliations.

Déclaration du numéro spécial. This article is part of the special issue "Hydrology of Large River Basins of Africa". It is a result of the 4th International Conference on the "Hydrology of the Great Rivers of Africa", Cotonou, Benin, 13-20 November 2021.

Remerciements. Les auteurs remercient le comité d'organisation du Conférence international sur les grands fleuves africains de nous avoir donné l'opportunité de publier cet article. Merci pour leurs contributions.

\section{Références}

Abossolo, S. A., Amougou, J. A., Tchindjang, M., Mena, M. S., et Batha, R. A. S. : Analyse des précipitations annuelles à la station de Yaoundé de 1895 à 2006, Afrique Science, 11, 183-194, 2015.

Ardoin-Bardin, S. : Variabilité hydroclimatique et impacts sur les ressources en eau de grands bassins hydrographiques en zone soudano-sahélienne. Thèse de doctorat, option mécanique, génie mécanique et génie civil, Université Montpellier II, Montpellier, 440 pp., 2004.

Barraud, G. : Caractérisation des saisons sèches dans les zones forestières d'Afrique Centrale : approche multi-base de données combinant télédétection et observations, Mémoire de Master SEME, Université de Bourgogne, Dijon, 50 pp., 2017.

Barron, J., Rockström, J., Gichuki, F., and Hatibu, N. : Dry spell analysis and maize yields for two semi-arid locations in east Africa, Agric. For. Meteorol., 117, 23-37, 2003.

Camberlin, P. : L'Afrique Centrale dans le contexte de la variabilité climatique tropicale interannuelle et intra-saisonnière, Presses Universitaires d'Orléans, L'Afrique Centrale, le Cameroun et les changements globaux, Yaoundé, 25-39, 2007.

Camberlin, P., Barraud, G., Bigot, S., Dewitte, O., Makanzu Imwangana, F., Maki Mateso, J.C., Martiny, N., Monsieurs, E., Moron, V., Pellarin, T., Philippon, N., Sahani, M., and Samba, G. : Evaluation of remotely sensed rainfall products over Central Africa, Q. J. Roy. Meteor. Soc., 145, 2115-2138, 2019.

COMIFAC : Forêt d'Afrique centrale et changement climatique, $\mathrm{Pu}$ blication de l'Observatoire des forêts d'Afrique centrale, 2015.
GIEC : Rapport de synthèse. Contribution des Groupes de travail I, II et III au cinquième Rapport d'évaluation du Groupe d'experts intergouvernemental sur l'évolution du climat, Sous la direction de l'équipe de rédaction principale, édité par: Pachauri, R. K. et Meyer, L. A., GIEC, Genève, Suisse, 161 pp., 2014.

Janicot, S. et Sultan, B. : Intra-seasonal modulation of convection in the West African monsoon, Geophys. Res. Lett., 28, 523-527, 2001.

Lamb, P. : Subsaharan rainfall update for 1982 : continued drought, J. Climatol., 3, 419-422, 1983.

Liebmann, B., Bladé, I., Bond, N. A., Gochis, D., Allured, D., and Bates, G. T. : Characteristics of North American summertime rainfall with emphasis on the monsoon, J. Climate, 21, 12771294, 2008.

Liebmann, B., Bladé, I., Kiladis, G. N., Carvalho, L. M., Senay, D., and Allured, S. : Seasonality of African precipitation from 1996 to 2009, J. Climate, 25, 4304-4322, 2012.

Massouangui-Kifouala, M. : Caractérisation des saisons pluvioagricoles dans les pays de Niari, République du Congo, en : Géographie du Congo, mélanges offerts au professeur Bonaventure Mengho, L'Harmattan, Paris, 225-246, 2017.

Ndjéndolé, S. : Contraintes pluviométriques sur les cultures annuelles : l'exemple de la culture cotonnière. Thèse de doctorat de Géographie, option agro-climatologie, Université de Bourgogne, Dijon, 227 pp., 2001.

Pettitt, A. N. A. : Non parametric approach to the change point problem, Appl. Stat., 28, 126-135, 1979.

Raghavendra A., Zhou, L., Jiang, Y., and Wenjian, H. : Increasing extent and intensity of thunderstorms observed over the Congo Basin from 1982 to 2016, Atmos. Res., 213, 17-26, 2018.

Sanders, L. : L'analyse statistique des données géographiques, G.I.P RECLUS, Montpellier, 269 pp., 1989.

Taylor, C. M., Fink, A. H., Klein, C., Parker, D. J., Guichard, F., Harris, P. P., and Knapp, K. R. : Earlier seasonal onset of intense mesoscale convective systems in the Congo Basin since 1999, Geophys. Res. Lett., 45, 458-467, 2018.

World Meteorological Organization : Climate Explorer, disponible sur : https://climexp.knmi.nl/ (la date du dernier accès : 14 mars 2019), WMO [data set], 2019.

Zhou, L., Tian, Y., Myneni, R. B., Ciais, P., Saatchi, S., Liu, Y. Y., and Hwang, T. : Widespread decline of Congo rainforest greenness in the past decade, Nature, 509, 86, 2014. 